\title{
Research on Steam Flooding Technology in Deep Heavy Oil Reservoir Bin $\mathrm{Lu}^{1, \mathrm{a}}$ \\ ${ }^{1}$ China National Oil and Gas Exploration and Development Corporation, Beijing, China, 100034 ${ }^{a}$ email,
}

\author{
Keywords: Steam Flooding Technology, Deep Heavy Oil, Reservoir
}

\begin{abstract}
The majority of heavy oil block has entered a phase of high-throughput round in China, mining results have clearly deteriorated, The natural reservoir has been a marked lack of energy, resulting in reduced cycle of oil production, oil and gas than the lower mining costs, economic deterioration. The research and testing cost-effective to replace the cycle steam mining technology, to further enhance oil recovery is a main technical problems demanding prompt solution in heavy oil development.
\end{abstract}

\section{Introduction}

Heavy oil is account for a large proportion of the world's oil and gas resources. After twenty years of development, the block has a lot of heavy oil into the high throughput round stage, stimulation effect has obviously deteriorated mining, natural reservoir energy is obviously insufficient, leading to reduce cycle oil production, oil and gas ratio is reduced, rising mining costs, economic efficiency is degraded. Therefore, research and testing cost-effective to replace steam stimulation mining technology to further enhance oil recovery, is a major technical problem currently solved in heavy oil development. In fact, to further improve the recovery is a common problem faced by the various producers of heavy oil after steam stimulation. Steam flooding technology in China is still in the trial and the initial stage. Karamay nine districts shallow reservoirs larger steam flooding, steam flooding has invested more than 650 well groups, steam flooding yield of about $85 \times 104$ t, steam oil ratio of 0.2 , the operating costs are still high. Shengli Oilfield heavy oil block buried deep in the deep heavy oil reservoir steam flooding technology is still in an experimental stage, for various reasons, most of the blocks are not transferred to steam flooding mining. To solve the above problem, this paper mainly for research in deep heavy oil reservoir steam flooding steam injection parameters, derived mathematical model steam front for dynamic monitoring of steam drive propulsion azimuth and range spread, and the deep heavy oil reservoir steam drive economic feasibility evaluation.

\section{Mining Steam Flooding Mechanism}

Steam flooding method is used by the group consisting of injection and production wells flooding patterns, steam injection and production wells can be determinant well or well area network (five points, seven anti-anti-nine) cloth well. From the continuous injection of steam injection wells to drive oil to the production wells and heating oil will be in the process, reducing its viscosity. After a lot of indoor simulation study results show that the steam flooding mining mechanism including: (1) viscosity reduction; miscible oil; (2) thermal expansion; (3) distillation of steam; (4) degassing displacement effect; (5) was dissolved gas drive action; (6) emulsification flooding effect; (7) to increase the role of the oil relative permeability; (8) the role gravity separation. Which mechanism plays a leading role depends on the thermodynamic properties of the reservoir and the type of oil. For the oil reservoir, viscosity and steam distillation is the most important mechanism of flooding.

\section{Optimum Design of Steam Flooding}

Evaluation Procedures and Method for Selection of Steam Flooding Reservoir. Not all are suitable for heavy oil reservoir steam flooding, according to consider the economic recovery and 
reservoir parameters. Mainly to see the steam flooding compared with other possible ways of development can achieve if relatively high recovery and economic benefits. So the steam flooding reservoirs need to choose, and to assess the effect of steam flooding and economic development.

Reservoir parameters by quantitative research on the effect of steam flooding, as well as a large number of previous proposed screening criteria, China Petroleum Exploration and Development Research Institute proposed steam flooding screening criteria. It should be noted that the selection criteria is not absolute, it is just to play a guiding role. With the progress of science and technology, the screening criteria boundaries will become increasingly wide. Of conglomerate reservoir, porosity may be relaxed; throughput for the first warm-up reservoir, oil viscosity may be relaxed; for closing the reservoir, in the case of insulated tubing and efficient conditions, the depth may be relaxed.

In addition to the above parameters to consider the impact of steam flooding, it must also consider the lithology, reservoir pressure, formation dip, reservoir connectivity, reservoir vertical heterogeneity, affecting the top edge and bottom water and gas and other factors.

The selected reservoir steam flooding recovery forecast. According to well spacing density and optimal well pattern design, combined with the current status of the development of the reservoir, steam flooding determined the need to increase the number of wells and the number of boiler. This method results predicted reservoir conditions achievable recovery. Suitable for medium and high porosity and high permeability and less active edge and bottom water reservoir steam flooding recovery forecast.

Input-Output estimates was assessed steam flooding money. Steam flooding large investment, so before you apply this method must be carried out to assess the economic benefits, the use of methods to assess the input-output method estimates. First, the predicted steam drive stage before the recovery factor and production time. The sale of oil revenues during the production period deducting fuel, integrated tax, total operating expenses, which is under way continue to produce economic benefits, return on investment has been assumed here. Steam drive production period oil sales revenue minus the cost of fuel, the new investments, consolidated tax, total operating expenses is steam flooding money. Finally, the comparison does not turn drive and drive economic benefits in two ways under the steam can drive economic assessment. The absolute value of the deviation must be calculated economic benefit here, but the relative value is quite reliable.

Optimization of Steam Flooding Operations. Elected for steam flooding reservoir, steam flooding not necessarily guarantee success. Practice shows that the steam drive success, not only with the reservoir conditions, and also have a great relationship with the steam flooding operating conditions. Domestic and international experience and research shows that steam drive, steam drive to make the reservoir conditions to be met to achieve the steam flooding recovery, you must also meet the following four operating conditions of steam drive

Steam injection rate: $1.6-1.8 \mathrm{t} /(\mathrm{d} \bullet \mathrm{m} \bullet \mathrm{ha})$;

Note Mining ratio: $1.2-1.3$

Bottom hole steam dryness: $>40 \%$;

Reservoir pressure: $<5 \mathrm{MPa}$, preferably 1-3MPa.

Steam injection and production injection rate than the reason why there is a range, which is the reservoir conditions (depth, thickness ratio of total net reservoir pressure) and possible related dryness bottom.

\section{Research on Steam Flooding Technology Drilling}

Drilling Technology. For 40 relatively loose reservoir characteristics, and in order to ensure that the rules of well trajectory, the design of the surface casing drilling depths to $250 \mathrm{~m}$, the production casing made of high strength TP120TH, while taking completion. At the same time taking into account the rotation drive by the original reservoir pressure drops to the actual pressure of $815 \mathrm{MPa}$ case $2 \sim 3 \mathrm{MPa}$, in order to prevent oil pollution, the use of high-quality drilling fluids, drilling mud invasion depth is $40 \mathrm{~mm}$ down to $15 \mathrm{~mm}$, greatly reducing the drilling fluid contamination reservoir. 
Steam Injection Process. Ground steam injection pipeline porous calcium silicate insulation materials, and taking into account the reduction in the thickness of the insulation layer tying designed insulation thickness of $50 \mathrm{~mm}$. Steam injection string consists of vacuum insulated pipe, insulation type coupling, pressure compensating insulation Steam flooding telescopic tube, Y441 steam flooding forced unblocking packer, high temperature single-valve, multi-stage steam drive long-term seal and other tools. Steam injection string insulated manner, through numerical simulation software to calculate, design uses: + vacuum insulated pipe insulation or packer annulus nitrogen injection insulated manner. Column temperature 360e, pressure $17 \mathrm{MPa}$, the service life of three years. Multilayer layered heavy oil reservoirs, in order to avoid the use of layers due to the extent of heterogeneity formation caused by imbalance, a hierarchical design steam drive processes. Through the ground simulation software and calculations to determine the sub-layers segments with steam and steam distribution apertures corresponding intervals, optimizing the design with the steam valve and pulling with steam in order to achieve the adjustment, to ensure the implementation of the hierarchical steam drive effectiveness.

Lifting Technique. Characteristics of different production fluid temperature for steam flooding during different stages of the research and development of non-metallic, metal, ceramics three categories series of lifting steam flooding technology, including high-temperature floating ring pumps, flexible metal pump, ceramic high-temperature steam drive wells pumps, steam drive to meet the extract needs to adapt to high temperature steam flooding well, complex well conditions sand, corrosion, high-containing gas.

High-Temperature Steam Flooding Snubbing. High temperature steam flooding technology snubbing operations research, to solve the well medium to high temperature steam conditions, do not take any measures to kill the high-temperature operation problems. OS Path 0-180 mm, temperature $260 \mathrm{e}$, pressure $21 \mathrm{MPa}$, adapt to the environment temperature $-40-50 \mathrm{e}$; the whole system is equipped with automatic and manual control, with on-site operation, to meet the construction and operation of the various systems from the next column force control requirements.

\section{Steam Flooding Experimental Tracking Research and Evaluation}

Pilot area consists of four $70 \mathrm{~m}$ well spacing group consisting of inverted nine wells, located in Block 8-c261 central well area. Test object layer lotus Ò oil group. Forward drive test area a total of nine production wells, the use of 141 square well spacing well pattern, cyclic steam stimulation mode mining, handling an average of 717 times, recovery of $24 \%$, the reservoir pressure drops to 3-4 MPa.

Analysis of the Production Characteristics. Its production features dynamic performance, thermal communication phase - transfer drive after the first half, increased fluid production, oil production decline. Flooding stage - the first three years, the amount of fluid production and oil production continued to rise after the transfer drive (210 m3 respectively, by that effect before / d and $30 \mathrm{t} / \mathrm{d}$ increased to $590 \mathrm{~m} 3 / \mathrm{d}$ and $154 \mathrm{t} / \mathrm{d}$ ) and maintain the basic stability of the instantaneous steam oil ratio of about 0122. Breakthrough stage - the first four years after the revolution drive, fluid production and oil production begins to decline.

Changes in reservoir temperature and pressure steam flooding are also consistent with the law. Test results from the temperature observation wells of steam injection wells 9-x26 wells $2715 \mathrm{~m}$, and oil temperature rise after drive. Rotation drive shaft 5 months was observed after the oil temperature rises sections $132 \mathrm{e}, 100 \mathrm{e}$ in which $51 \%$ or more of the thickness of the total thickness of the oil layer, and concentrated in the central reservoir. To stage the 2001 flooding, the reservoir has reached a temperature section 220e, 67\% 100e than the total thickness of the oil layer thickness, that is, the upper reservoir are heated, and the thickness of the heating increases. Wellhead production fluid temperature generally on the rise, driven by the right and left turn when 30e rises to 2001 65-100e, estimating bottom hole temperature should be around 140-180e. Pressure data indicates that the forwarding drives load $411 \mathrm{MPa}$, and then gradually decreased to 315MPa. Steam flooding is the ideal pressure range.

Adjustment Tracking Study. In thermal communication stage, steam injection well bore heat 
loss, the initial bottom hole dryness does not meet program requirements. To reduce heat loss, to fill the annulus regular nitrogen, reducing heat loss to improve the bottom steam dryness. Pilot test of steam injection wells in December 1998 in a row three times up nitrogen and the down hole steam dryness increase from 3310\% to 50\%. Meanwhile, for the stage in thermal communication with low reservoir level, the problem of insufficient oil supply was carried out six wells cited throughput efficiency. After the stimulation lead effect, set well fluid production continues to rise, oil rebounded, rising by-turn driving early $29 \mathrm{t} / \mathrm{d}$ to $70 \mathrm{t} / \mathrm{d}$.

In a breakthrough stage, steam flooding late wellhead temperature to rise sharply fluid production and the sharp drop in oil production appears, for production wells conducted high temperature profile seal channeling technology, large diameter sealing technology, ease the steam channeling a certain extent problem. At the same time by optimizing the tracking numerical simulation analysis, taken to reduce the amount of steam injection practices, effectively inhibited the steam coming into and improve the ratio of oil and gas wells Group. In December 2001, May 2002, the group of well steam injection rate was adjusted twice, oil and gas wells group ratio remained at 0114.2003 November test wells group the third adjustment, stop Note 2 steam injection wells, steam injection wells set daily amount increased from $540 \mathrm{t} / \mathrm{d}$ was adjusted to $260 \mathrm{t} / \mathrm{d}$, oil production did not appear larger decline, remained at $50 \mathrm{t} / \mathrm{d}$ level of around 0120 over oil and gas about.

Evaluation. Pilot test achieved good development results. From January 1998 to June 2008, tired of steam injection pilot area $15217 * 104 \mathrm{t}$, the cumulative oil production $27148 * 104 \mathrm{t}$, more than 01 oil and gas phases, phase recovery percent 3119 percent, throughput $+5519 \%$ recovery steam drive, over design recovery (4815\%). Trial is still in progress, the current production rate is still at $118 \%$ of the level of recovery is expected to eventually reach $63 \%$.

Expand the pilot test area and test area adjacent to the reservoir conditions are generally similar, but slightly smaller than the thickness of the oil layer. Pilot area consists of seven $70 \mathrm{~m}$ well spacing group consisting of anti-nine wells. Test object layer lotus Ò oil group. Test area bearing area 01208 $\mathrm{km} 2$, original geological reserves $128106 * 104 \mathrm{t}$.

Expand the test began in July 2003. Turn drive throughput former mining recovery factor of 29\%, $53 \%$ of the initial oil saturation and reservoir pressure of about $2 \mathrm{MPa}$. By the end of June 2008, to expand the trials have been carried out for five years, steam flooding can $1315 \%$. And the comparison between design, program design and the actual steam injection quantity indicators remained the same, but oil production, fluid production, oil and gas ratio, production injection ratio and other indicators and design specifications there are some differences, mainly due to the spillover Pilot Test influence, forwarding flooding and high degree of factors.

\section{Conclusion}

Late in the deep heavy oil reservoir exploitation of most blocks have been developed in throughput, the prevalence of poor mining, mining is difficult problem. Steam flooding pilot test is completed successfully and promotion for future large-scale transfer drive to improve heavy oil recovery, provide the necessary technical support and practical experience.

\section{References}

[1] Li Jingyuan. Southwest Petroleum University, Vol. 6 (2014) No 53, p.25-26

[2] Wang Qunyong. Special Reservoirs, Vol. 12 (2015) No 27, p.74-76

[3] Jing Jianfen, Hou XuSiem. Foreign Oil Field Engineering, Vol. 30 (2014) No 19, p.144-145

[4] Wang Kuailiang. China Offshore Oil and Gas Geology, Vol. 29 (2010) No 27, p.21-23

[5] Zhang Gongxu, Sun Jing. Special Reservoirs, Vol. 8 (2013) No 27, p.57-60 